\title{
Accurate nucleon-nucleon potential with charge-independence breaking
}

\author{
R. B. Wiringa \\ Physics Division, Argonne National Laboratory, Argonne, Illinois 60439 \\ V. G. J. Stoks \\ School of Physical Sciences, The Flinders University of South Australia, Bedford Park, South Australia 5042, Australia \\ R. Schiavilla \\ CEBAF Theory Group, Newport News, Virginia 23606 \\ and Department of Physics, Old Dominion University, Norfolk, Virginia 23529
}

(Received 15 August 1994)

\begin{abstract}
We present a new high-quality nucleon-nucleon potential with explicit charge dependence and charge asymmetry, which we designate Argonne $v_{18}$. The model has a charge-independent part with 14 operator components that is an updated version of the Argonne $v_{14}$ potential. Three additional charge-dependent and one charge-asymmetric operators are added, along with a complete electromagnetic interaction. The potential has been fit directly to the Nijmegen $p p$ and $n p$ scattering database, low-energy $n n$ scattering parameters, and deuteron binding energy. With 40 adjustable parameters it gives a $\chi^{2}$ per datum of 1.09 for $4301 p p$ and $n p$ data in the range 0-350 $\mathrm{MeV}$.
\end{abstract}

PACS number(s): 13.75.Cs, 12.39.Pn, 21.30.+y

\section{INTRODUCTION}

Traditionally, nucleon-nucleon $(N N)$ potentials are constructed by fitting $n p$ data for $T=0$ states and either $n p$ or $p p$ data for $T=1$ states. Examples of potentials fit to $n p$ data in all states are the Argonne $v_{14}[1]$, Urbana $v_{14}[2]$, and most of the Bonn potentials [3,4]. In contrast, the Reid [5], Nijmegen [6], and Paris [7] potentials were fit to $p p$ data for $T=1$ channels. Unfortunately, potential models which have been fit only to the $n p$ data often give a poor description of the $p p$ data [8], even after applying the necessary corrections for the Coulomb interaction. By the same token, potentials fit to $p p$ data in $T=1$ states give only a mediocre description of $n p$ data. Fundamentally, this problem is due to charge-independence breaking in the strong interaction.

In the present work we construct an updated version of the Argonne potential that fits both $p p$ and $n p$ data, as well as low-energy $n n$ scattering parameters and deuteron properties. The strong interaction potential is written in an operator format that depends on the values of $S, T$, and $T_{z}$ of the $N N$ pair. We then project the potential into a charge-independent (CI) part that has 14 operator components (as in the older Argonne $v_{14}$ model) and a charge-independence breaking (CIB) part that has three charge-dependent (CD) and one charge-asymmetric (CA) operators. We also include a complete electromagnetic potential, containing Coulomb, Darwin-Foldy, vacuum polarization, and magnetic moment terms with finite-size effects. We designate the new model Argonne $v_{18}$.

In a number of applications it is important for a $N N$ potential to reproduce correct $n p$ and $p p$ scattering parameters. For example, in thermal neutron radiative capture on the proton, ${ }^{1} \mathrm{H}(n, \gamma)^{2} \mathrm{H}$, it is crucial to have the correct singlet $n p$ scattering length in the initial state to get the cross section. However, in low-energy proton weak capture, ${ }^{1} \mathrm{H}\left(p, e^{+} \nu_{e}\right)^{2} \mathrm{H}$, it is equally important that the correct $p p$ scattering length be provided by the interaction. Clearly, a complete potential model should meet both requirements.

Another important application is in the formulation of three-nucleon $(N N N)$ potentials. In general, nuclei are underbound using only $N N$ potentials fit to the scattering data. Nontrivial many-nucleon interactions are expected to make up a portion of the missing binding energy. Phenomenologically we may choose to construct a many-body Hamiltonian, such as

$$
H=\sum_{i} \frac{-\hbar^{2}}{2 M_{i}} \nabla_{i}^{2}+\sum_{i<j} v_{i j}+\sum_{i<j<k} V_{i j k}
$$

and constrain the strength parameters of the $N N N$ potential by requiring that $H$ gives the correct trinucleon binding energy. Similar considerations apply if we choose a relativistic formulation. Clearly, such constraints are ambiguous or even meaningless if the $N N$ potential used in the calculations does not adequately describe the twonucleon data. For ${ }^{3} \mathrm{He}\left({ }^{3} \mathrm{H}\right)$, in which the $N N$ interaction underbinds by $\sim 1 \mathrm{MeV}$, there are two $n p$ pairs and one $p p(n n)$ pair. To a good approximation, the two $n p$ pairs will be in the $S=1, T=0$ state $75 \%$ of the time, and in the $S=0, T=1$ state $25 \%$ of the time, while the $p p$ ( $n n$ ) pair will be pure $S=0, T=1$. If the chosen $N N$ potential fits only the more repulsive $p p(n n)$ data in the $T=1$ state, we would get a smaller $N N$ contribution to the binding energy and thus overestimate the $N N N$ potential strength required. By the same token, a model fit to $n p$ data in the $T=1$ state would be too attractive and we would underestimate the $N N N$ potential. The difference can be as much as $0.4 \mathrm{MeV}$, leading to variations in 
the $N N N$ potential strength of order $\pm 20 \%$. This would have significant effects in larger many-body systems.

Because we include a complete electromagnetic potential and fit low-energy $n n$ scattering parameters, the present model also can be used to study charge-symmetry breaking, as in the ${ }^{3} \mathrm{H}-{ }^{3} \mathrm{He}$ mass difference [9], or more generally the Nolen-Schiffer anomaly [10]. The electromagnetic potential is in principle well known and is the longest-range part of the interaction. Potential models commonly fit the deuteron energy to better than $1 \mathrm{keV}$ accuracy. Since we find that the electromagnetic terms give a non-negligible $18 \mathrm{keV}$ repulsion in the deuteron and moderate shifts in the $n p$ and $n n$ scattering lengths, we deem it desirable to include these terms explicitly.

The major goal of the present work is to construct a nonrelativistic potential that can be used easily in nuclear many-body calculations and that accurately fits both $p p$ and $n p$ data. We adopt the local operator structure of the older Argonne $v_{14}$ and Urbana $v_{14}$ potentials, which have been used extensively in calculations of finite nuclei, nuclear matter, and neutron stars [11-13]. The assumption of an underlying operator structure relates all partial waves in a simple manner, without imposing a oneboson-exchange (OBE) form which might be too restrictive at short distances. Recently, the Nijmegen group has shown [14] that it is feasible to construct potential models which fit the $N N$ data with the almost perfect $\chi^{2}$ per datum of 1 . However, these models differ in each partial wave and thus implicitly introduce nonlocalities from one partial wave to the next that may be difficult to characterize and treat accurately in many-body calculations. When they limit the potential to an OBE form, which has a local operator structure (save for a nonlocal part in the central potential) describing all partial waves simultaneously, the $\chi^{2}$ per datum increases to 1.87 , albeit with a much smaller number of parameters. The present model is a compromise between these two approaches, adopting a phenomenological form (unrestricted by an OBE picture) at short distances, but maintaining a local operator structure. The potential was directly fit to the Nijmegen $N N$ scattering database $[15,16]$, which contains $1787 p p$ and $2514 n p$ data in the range $0-350 \mathrm{MeV}$, and has an excellent $\chi^{2}$ per datum of 1.09 .

In Sec. II we present the analytical form of the potential in the various spin and isospin states. Special attention is given to the electromagnetic part of the interaction. The free parameters are fit to the $N N$ scattering data and deuteron binding energy in Sec. III, where we also present the phase shifts. Section IV discusses the projection of the potential into operator format. Static deuteron properties and electromagnetic form factors, with relativisitic and exchange current contributions, are presented in Sec. V. Conclusions and an outlook are given in Sec. VI.

\section{FORM OF THE POTENTIAL IN $S, T, T_{z}$ STATES}

The $N N$ potential is written as a sum of an electromagnetic (EM) part, a one-pion-exchange (OPE) part, and an intermediate- and short-range phenomenological part:

$$
v(N N)=v^{\mathrm{EM}}(N N)+v^{\pi}(N N)+v^{R}(N N) .
$$

The EM interaction is the same as that used in the Nijmegen partial-wave analysis, with the addition of short-range terms and finite-size effects [17-19]. (Values for the masses and other physical constants used in the following formulae are given in Table I.) For $p p$ scattering we include one- and two-photon Coulomb terms, the Darwin-Foldy term, vacuum polarization, and the magnetic moment interaction, each with an appropriate form factor:

$$
v^{\mathrm{EM}}(p p)=V_{C 1}(p p)+V_{C 2}+V_{D F}+V_{V P}+V_{M M}(p p) .
$$

Here

$$
\begin{aligned}
& V_{C 1}(p p)=\alpha^{\prime} \frac{F_{C}(r)}{r}, \\
& V_{C 2}=-\frac{\alpha}{2 M_{p}^{2}}\left[\left(\nabla^{2}+k^{2}\right) \frac{F_{C}(r)}{r}+\frac{F_{C}(r)}{r}\left(\nabla^{2}+k^{2}\right)\right] \approx-\frac{\alpha \alpha^{\prime}}{M_{p}}\left[\frac{\left.F_{C}(r)\right]^{2}}{r}\right]^{2} \\
& V_{D F}=-\frac{\alpha}{4 M_{p}^{2}} F_{\delta}(r) \\
& V_{V P}=\frac{2 \alpha \alpha^{\prime}}{3 \pi} \frac{F_{C}(r)}{r} \int_{1}^{\infty} d x e^{-2 m_{e} r x}\left[1+\frac{1}{2 x^{2}}\right] \frac{\left(x^{2}-1\right)^{1 / 2}}{x^{2}}, \\
& V_{M M}(p p)=-\frac{\alpha}{4 M_{p}^{2}} \mu_{p}^{2}\left[\frac{2}{3} F_{\delta}(r) \sigma_{i} \cdot \sigma_{j}+\frac{F_{t}(r)}{r^{3}} S_{i j}\right]-\frac{\alpha}{2 M_{p}^{2}}\left(4 \mu_{p}-1\right) \frac{F_{l s}(r)}{r^{3}} \mathbf{L} \cdot \mathbf{S} .
\end{aligned}
$$


TABLE I. Values of fundamental constants adopted in this work.

\begin{tabular}{crl}
\hline \hline$\hbar c$ & 197.32705 & $\mathrm{MeVfm}$ \\
$m_{\pi^{0}}$ & 134.9739 & $\mathrm{MeV} / c^{2}$ \\
$m_{\pi^{ \pm}}$ & 139.5675 & $\mathrm{MeV} / c^{2}$ \\
$M_{p}$ & 938.27231 & $\mathrm{MeV} / c^{2}$ \\
$M_{n}$ & 939.56563 & $\mathrm{MeV} / c^{2}$ \\
$\alpha^{-1}$ & 137.03599 & \\
$\mu_{p}$ & 2.79285 & $\mu_{0}$ \\
$\mu_{n}$ & -1.91304 & $\mu_{0}$ \\
\hline \hline
\end{tabular}

The Coulomb interaction includes an energy dependence through the $\alpha^{\prime} \equiv 2 k \alpha /\left(M_{p} v_{\text {lab }}\right)$ [20], which is significantly different from $\alpha$ at even moderate energies $(\sim 20 \%$ difference at $T_{\text {lab }}=250 \mathrm{MeV}$ ). The vacuum polarization and two-photon Coulomb interaction are important for fitting the high-precision low-energy scattering data. The $F_{C}, F_{\delta}, F_{t}$, and $F_{l s}$ are short-range functions that represent the finite size of the nucleon charge distributions. They have been obtained under the assumption that the nucleon form factors are well represented by a dipole form

$$
G_{E}^{p}=\frac{G_{M}^{p}}{\mu_{p}}=\frac{G_{M}^{n}}{\mu_{n}}=G_{D}=\left(1+\frac{q^{2}}{b^{2}}\right)^{-2}
$$

where $b=4.27 \mathrm{fm}^{-1}$. The functions are given by

$$
\begin{aligned}
& F_{C}(r)=1-\left(1+\frac{11}{16} x+\frac{3}{16} x^{2}+\frac{1}{48} x^{3}\right) e^{-x} \\
& F_{\delta}(r)=b^{3}\left(\frac{1}{16}+\frac{1}{16} x+\frac{1}{48} x^{2}\right) e^{-x} \\
& F_{t}(r)=1-\left(1+x+\frac{1}{2} x^{2}+\frac{1}{6} x^{3}+\frac{1}{24} x^{4}+\frac{1}{144} x^{5}\right) e^{-x} \\
& F_{l s}(r)=1-\left(1+x+\frac{1}{2} x^{2}+\frac{7}{48} x^{3}+\frac{1}{48} x^{4}\right) e^{-x}
\end{aligned}
$$

with $x=b r$. The derivation of $F_{C}$ is given in [21], while the others are related by $F_{\delta}=-\nabla^{2}\left(F_{C} / r\right), F_{t}=$ $\left(F_{C} / r\right)^{\prime \prime}-\left(F_{C} / r\right)^{\prime} / r$, and $F_{l s}=\left(F_{C} / r\right)^{\prime} / r$. In the limit of point nucleons, $F_{C}=F_{t}=F_{l s}=1$ and $F_{\delta}=4 \pi \delta^{3}(\mathbf{r})$. These form factors are illustrated in Fig. 1. The use of $F_{C}$ in $V_{V P}$ is an approximate method of removing the $1 / r$ singularity (the logarithmic singularity remains) which is justified by its short range and the overall smallness of the term. Similarly, the use of $F_{C}^{2}$ in $V_{C 2}$ is an approximate method of removing the $1 / r^{2}$ singularity. We note that because we use the Sachs nucleon form factors, there are no additional magnetic Darwin-Foldy terms [22].

For the $n p$ system we include a Coulomb term attributable to the neutron charge distribution in addition to the interaction between magnetic moments,

$$
v^{\mathrm{EM}}(n p)=V_{C 1}(n p)+V_{M M}(n p) .
$$

Here

$$
V_{C 1}(n p)=\alpha \beta_{n} \frac{F_{n p}(r)}{r}
$$

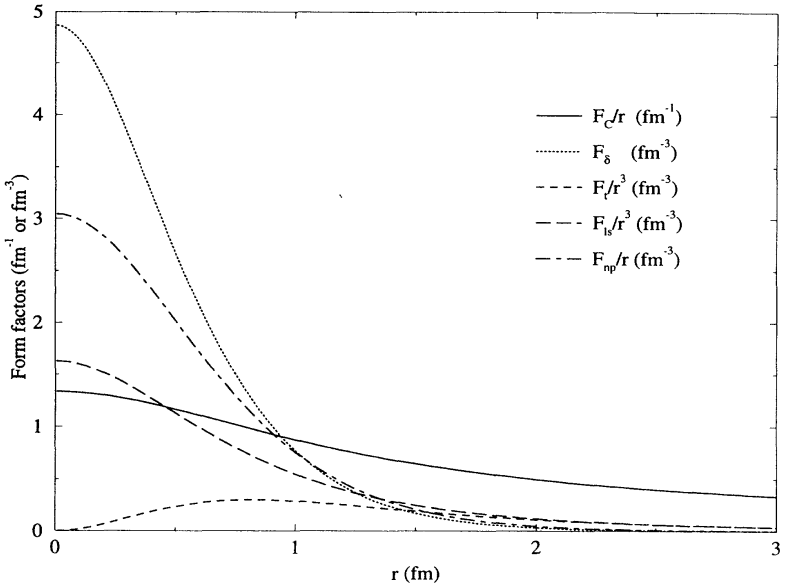

FIG. 1. Form factors in the electromagnetic interaction.

where the function $F_{n p}$ is obtained assuming the neutron electric form factor [22]

$$
G_{E}^{n}=\beta_{n} q^{2}\left(1+\frac{q^{2}}{b^{2}}\right)^{-3} .
$$

Here $\beta_{n} \equiv\left[d G_{E}^{n} / d q^{2}\right]_{q=0}=0.0189 \mathrm{fm}^{2}$, the experimentally measured slope [23]. We have checked this form factor in a self-consistent calculation of the deuteron structure function $A\left(q^{2}\right)$ used to extract $G_{E}^{n}$ [24] and find it gives a fairly good fit to the data. This simple form leads to

$$
F_{n p}(r)=b^{2}\left(15 x+15 x^{2}+6 x^{3}+x^{4}\right) \frac{e^{-x}}{384} .
$$

The $F_{n p}$ is also shown in Fig. 1. The magnetic moment interaction is given by

$$
\begin{aligned}
V_{M M}(n p)= & -\frac{\alpha}{4 M_{n} M_{p}} \mu_{n} \mu_{p}\left[\frac{2}{3} F_{\delta}(r) \sigma_{i} \cdot \boldsymbol{\sigma}_{j}+\frac{F_{t}(r)}{r^{3}} S_{i j}\right] \\
& -\frac{\alpha}{2 M_{n} M_{r}} \mu_{n} \frac{F_{l s}(r)}{r^{3}}(\mathbf{L} \cdot \mathbf{S}+\mathbf{L} \cdot \mathbf{A})
\end{aligned}
$$

where $M_{r}$ is the nucleon reduced mass. The term proportional to $\mathbf{A}=\frac{1}{2}\left(\sigma_{i}-\sigma_{j}\right)$ is a "class IV" chargeasymmetric force [25], which mixes spin-singlet and spintriplet states. Its contribution is very small, and we only include it when we construct the magnetic moment scattering amplitude [19].

Finally, for $n n$ scattering, we neglect the Coulomb interaction between the neutron form factors, so there is only a magnetic moment term

$$
\begin{aligned}
v^{\mathrm{EM}}(n n) & =V_{M M}(n n) \\
& =-\frac{\alpha}{4 M_{n}^{2}} \mu_{n}^{2}\left[\frac{2}{3} F_{\delta}(r) \sigma_{i} \cdot \sigma_{j}+\frac{F_{t}(r)}{r^{3}} S_{i j}\right] .
\end{aligned}
$$

The charge-dependent structure of the OPE potential is the same as that used in the Nijmegen partial-wave analysis and reads 


$$
\begin{aligned}
& v^{\pi}(p p)=f_{p p}^{2} v_{\pi}\left(m_{\pi^{0}}\right) \\
& v^{\pi}(n p)=f_{p p} f_{n n} v_{\pi}\left(m_{\pi^{0}}\right)+(-)^{T+1} 2 f_{c}^{2} v_{\pi}\left(m_{\pi^{ \pm}}\right) \\
& v^{\pi}(n n)=f_{n n}^{2} v_{\pi}\left(m_{\pi^{0}}\right)
\end{aligned}
$$

where $T$ is the isospin and

$$
v_{\pi}(m)=\left(\frac{m}{m_{s}}\right)^{2} \frac{1}{3} m c^{2}\left[Y_{\mu}(r) \sigma_{i} \cdot \sigma_{j}+T_{\mu}(r) S_{i j}\right]
$$

(Strictly speaking, the neutron-proton mass difference gives rise to an OPE "class IV" force as well, which again we only explicitly include when we construct the OPE scattering amplitude [19].) Here $Y_{\mu}(r)$ and $T_{\mu}(r)$ are the usual Yukawa and tensor functions with the exponential cutoff of the Urbana and Argonne $v_{14}$ models

$$
\begin{aligned}
& Y_{\mu}(r)=\frac{e^{-\mu r}}{\mu r}\left(1-e^{-c r^{2}}\right) \\
& T_{\mu}(r)=\left(1+\frac{3}{\mu r}+\frac{3}{(\mu r)^{2}}\right) \frac{e^{-\mu r}}{\mu r}\left(1-e^{-c r^{2}}\right)^{2}
\end{aligned}
$$

where $\mu=m c / \hbar$. The scaling mass $m_{s}$, introduced in Eq. (18) to make the coupling constant dimensionless, is taken to be the charged-pion mass, $m_{\pi^{ \pm}}$. The Nijmegen partial-wave analysis of $N N$ scattering data below 350 $\mathrm{MeV}$ finds very little difference between the coupling constants [26], so we choose them to be charge independent, i.e., $f_{p p}=-f_{n n}=f_{c} \equiv f$, with the recommended value $f^{2}=0.075$. Thus all charge dependence in Eqs. (17) is due simply to the difference in the charged- and neutralpion masses.

The remaining intermediate- and short-range phenomenological part of the potential is expressed, as in the Argonne $v_{14}$ model, as a sum of central, $L^{2}$, tensor, spin-orbit, and quadratic spin-orbit terms (abbreviated as $c, l 2, t, l s, l s 2$, respectively) in different $S, T$, and $T_{z}$ states:

$$
v_{S T}^{R}(N N)=v_{S T, N N}^{c}(r)+v_{S T, N N}^{l 2}(r) L^{2}+v_{S T, N N}^{t}(r) S_{12}+v_{S T, N N}^{l s}(r) \mathbf{L} \cdot \mathbf{S}+v_{S T, N N}^{l_{s} 2}(r)(\mathbf{L} \cdot \mathbf{S})^{2} .
$$

Each of these terms is given the general form

$$
v_{S T, N N}^{i}(r)=I_{S T, N N}^{i} T_{\mu}^{2}(r)+\left[P_{S T, N N}^{i}+\mu r Q_{S T, N N}^{i}+(\mu r)^{2} R_{S T, N N}^{i}\right] W(r),
$$

where $\mu=\frac{1}{3}\left(m_{\pi^{0}}+2 m_{\pi^{ \pm}}\right) c / \hbar$ is the average of the pion masses and $T_{\mu}(r)$ is given by Eq. (19). Thus the $T_{\mu}^{2}(r)$ term has the range of a two-pion-exchange force. The $W(r)$ is a Woods-Saxon function which provides the short-range core:

$$
W(r)=\left[1+e^{\left(r-r_{0}\right) / a}\right]^{-1}
$$

The four sets of constants $I_{S T, N N}^{i}, P_{S T, N N}^{i}, Q_{S T, N N}^{i}$, and $R_{S T, N N}^{i}$ are parameters to be fit to data. However, we also impose a regularization condition at the origin which reduces the number of free parameters by one for each $v_{S T, N N}^{i}$. We require that

$$
\begin{gathered}
v_{S T, N N}^{t}(r=0)=0 \\
\left.\frac{\partial v_{S T, N N}^{i \neq t}}{\partial r}\right|_{r=0}=0 .
\end{gathered}
$$

Since the tensor part of the OPE potential already vanishes at $r=0$, the first condition is satisfied by setting $P_{S T, N N}^{t}=0$. The second condition is equivalent to fixing, for $i \neq t$,

$$
Q_{S T, N N}^{i}=-\frac{1}{\mu W(0)}\left[P_{S T, N N}^{i} \frac{\partial W}{\partial r}+\delta_{i c} \frac{\partial v_{S T}^{\pi}}{\partial r}\right]_{r=0}
$$

where we only have to evaluate the derivative of the spinspin part of the OPE potential.

\section{DATA FITTING}

An initial survey of possible potential forms was made by fitting to the $\chi^{2}$ hypersurface of the Nijmegen partialwave analysis of $p p$ and $n p$ data [16]. These studies helped select the final form of the potential ( $\sim 10$ variations were tried) and the values of the function shape parameters $c, r_{0}$, and $a$. Eventually, the cutoff parameter in the OPE functions $Y_{\mu}(r)$ and $T_{\mu}(r)$ was set at $c=2.1 \mathrm{fm}^{-2}$, while the parameters in the short-range Woods-Saxon $W(r)$ were set at $r_{0}=0.5 \mathrm{fm}$ and $a=0.2$ fm. This value of $c$ is slightly different from the $2.0 \mathrm{fm}^{-2}$ used in the Urbana and Argonne $v_{14}$ models, while $r_{0}$ and $a$ are the same. Attempts to make a softer-core model led to a poorer fit. Sensitivity to the OPE coupling constant was also checked before the recommended value $[26], f^{2}=0.075$, was adopted as satisfactory.

Once these four parameters were set, a preliminary fit of the remaining parameters $I_{S T, N N}^{i}, P_{S T, N N}^{i \neq t}, Q_{S T, N N}^{t}$, and $R_{S T, N N}^{i}$ to the phase shifts was made. The final values were obtained by a direct fit to the Nijmegen $p p$ and $n p$ scattering data base and the deuteron binding energy. We use nonrelativistic kinematics, i.e., the deuteron binding energy is taken as $E_{d}=\kappa^{2} / 2 M_{\mathrm{r}}$. In practice, we found no benefit to including an $R_{S T, N N}^{i}$ in spin-singlet states, so these values were set to zero. Also, we found no indication of a need for charge dependence in the phenomenological part of spin-triplet states. In the final fit 
TABLE II. Short-range potential parameters in MeV. The asterisk denotes that the value was computed by Eq. (23) and not fit. The three shape parameters are $c=2.1 \mathrm{fm}^{-2}, r_{0}=0.5 \mathrm{fm}$, and $a=0.2 \mathrm{fm}$.

\begin{tabular}{cccrrr}
\hline \hline Channel & Type & \multicolumn{1}{c}{$I$} & \multicolumn{1}{c}{$P$} & \multicolumn{1}{c}{$Q$} \\
\hline$S=0, T=1(p p)$ & $c$ & -11.27028 & 3346.6874 & $1859.5627 *$ & 0 \\
$S=0, T=1(n p)$ & $c$ & -10.66788 & 3126.5542 & $1746.4298 *$ & 0 \\
$S=0, T=1(n n)$ & $c$ & -11.27028 & 3342.7664 & $1857.4367 *$ & 0 \\
$S=0, T=1$ & $l 2$ & 0.12472 & 16.7780 & $9.0972 *$ & 0 \\
$S=0, T=0$ & $c$ & & & \\
& $l 2$ & -2.09971 & 1204.4301 & $511.9380 *$ & 0 \\
& & -0.31452 & 217.4559 & $117.9063 *$ & 0 \\
$S=1, T=1(p p)$ & $c$ & & & \\
$S=1, T=1(n p)$ & $c$ & -7.62701 & 1815.4920 & $969.3863 *$ & 1847.8059 \\
$S=1, T=1(n n)$ & $c$ & -7.62701 & 1813.5315 & $966.2483 *$ & 1847.8059 \\
$S=1, T=1$ & $l 2$ & -7.62701 & 1811.5710 & $967.2603 *$ & 1847.8059 \\
& $t$ & 0.06709 & 342.0669 & $185.4713 *$ & -615.2339 \\
& $l s$ & 1.07985 & 0 & -190.0949 & -811.2040 \\
& $l s 2$ & -0.62697 & -570.5571 & $-309.3605 *$ & 819.1222 \\
& & 0.74129 & 9.3418 & $5.0652 *$ & -376.4384 \\
$S=1, T=0$ & $c$ & & & & \\
& $l 2$ & -8.62770 & 2605.2682 & $1459.6345 *$ & 441.9733 \\
& $t$ & -0.13201 & 253.4350 & $137.4144 *$ & -1.0076 \\
& $l s$ & 1.485601 & 0 & -1126.8359 & 370.1324 \\
& $l s 2$ & 0.10180 & 86.0658 & $46.6655 *$ & -356.5175 \\
& & 0.07357 & -217.5791 & $-117.9731 *$ & 18.3935 \\
\hline \hline
\end{tabular}

there are 40 nonzero intermediate- and short-range parameters. These are given in Table II.

In addition, we fit the singlet $n n$ scattering length and effective range by slightly modifying the short-range $S=0, T=1 p p$ potential. The $n n$ effective range parameters are the only real experimental constraint on the $n n$ interaction, and they are not known very precisely. In particular, there is a considerable spread of values for ${ }^{1} a_{n n}$ depending on whether the experiment involves two or three nucleons in the final state [27]. We fit the values obtained from the analysis [28] of ${ }^{2} \mathrm{H}\left(\pi^{-}, \gamma\right) n n$ experiments [29,30], which are unaffected by possible three-nucleon forces and allow for a better determination of ${ }^{1} r_{n n}$. (They are also more consistent with the observed charge-symmetry breaking in the trin- ucleon bound state [9] and theoretical models based on $\rho-\omega$ and $\pi-\eta-\eta^{\prime}$ mixing [36].) This results in a small difference between $P_{01, p p}^{c}$ and $P_{01, n n}^{c}$. The same difference was carried over to the triplet channel by introducing a $P_{11, n n}^{c}$ term, as discussed below in Sec. IV. These additional potential parameters are also given in Table II.

The Nijmegen $N N$ scattering data base $[15,16]$ includes $1787 p p$ data (1656 observables and 131 normalization data) and $2514 n p$ data (2366 observables and 148 normalization data) in the range $0-350 \mathrm{MeV}$. The total $\chi^{2}$ of the potential is 4675 , divided into $\chi^{2}(p p)=1962$ and $\chi^{2}(n p)=2713$. A detailed breakdown of the $\chi^{2}$, analogous to Ref. [8] for the $p p$ data, is given in Table III. We also show the breakdown for the Nijmegen combined partial-wave analysis [16]. The difference between these

TABLE III. Distribution of $\chi^{2}$ by laboratory kinetic energy of the Nijmegen combined partial-wave analysis [16] (PWA93) and the new Argonne $v_{18}$ potential. $N_{p p}\left(N_{n p}\right)$ denotes the number of $p p(n p)$ data in each energy bin.

\begin{tabular}{|c|c|c|c|c|c|c|}
\hline \multirow[b]{2}{*}{$\operatorname{Bin}(\mathrm{MeV})$} & \multicolumn{4}{|c|}{$\chi^{2}(p p)$} & \multicolumn{2}{|c|}{$\chi^{2}(n p)$} \\
\hline & $N_{p p}$ & PWA93 & $v_{18}$ & $N_{n p}$ & PWA93 & $v_{18}$ \\
\hline $0.0-0.5$ & 134 & 134.5 & 136.3 & 10 & 9.7 & 11.8 \\
\hline $0.5-2$ & 63 & 39.7 & 41.1 & 5 & 3.8 & 7.4 \\
\hline $2-8$ & 48 & 45.0 & 36.0 & 55 & 52.4 & 51.0 \\
\hline $8-17$ & 108 & 103.0 & 111.6 & 182 & 168.3 & 164.8 \\
\hline $17-35$ & 59 & 63.1 & 72.2 & 293 & 226.6 & 234.9 \\
\hline $35-75$ & 243 & 213.4 & 251.5 & 328 & 335.2 & 339.3 \\
\hline $75-125$ & 167 & 169.5 & 171.5 & 232 & 237.1 & 231.3 \\
\hline $125-183$ & 343 & 379.7 & 415.7 & 333 & 336.8 & 363.5 \\
\hline $183-290$ & 239 & 285.9 & 304.8 & 517 & 494.6 & 574.0 \\
\hline $290-350$ & 383 & 360.7 & 421.3 & 571 & 599.0 & 708.0 \\
\hline $0-350$ & 1787 & 1794.5 & 1962.0 & 2526 & 2463.5 & 2685.8 \\
\hline
\end{tabular}


TABLE IV. $p p$ phase shifts in degrees. Energies are in MeV. The ${ }^{1} S_{0}$ includes the full electromagnetic interaction $\left[v^{\mathrm{EM}}(p p)\right]$ and is with respect to electromagnetic wave functions. The non- $S$ waves are nuclear phase shifts of the Coulomb interaction including the form factor $\left(V_{C 1}\right)$ with respect to Coulomb wave functions.

\begin{tabular}{rcccccccc}
\hline \hline$T_{\text {lab }}$ & \multicolumn{1}{c}{${ }^{1} S_{0}$} & ${ }^{1} D_{2}$ & ${ }^{3} P_{0}$ & ${ }^{3} P_{1}$ & ${ }^{3} P_{2}$ & $\varepsilon_{2}$ & ${ }^{3} F_{2}$ & ${ }^{3} F_{3}$ \\
\hline 1 & 32.68 & 0.00 & 0.14 & -0.08 & 0.01 & -0.00 & 0.00 & -0.00 \\
5 & 54.74 & 0.04 & 1.61 & -0.90 & 0.22 & -0.05 & 0.00 & -0.01 \\
10 & 55.09 & 0.17 & 3.80 & -2.05 & 0.66 & -0.20 & 0.01 & -0.03 \\
25 & 48.51 & 0.71 & 8.78 & -4.89 & 2.49 & -0.83 & 0.10 & -0.23 \\
50 & 38.78 & 1.73 & 11.75 & -8.23 & 5.79 & -1.77 & 0.32 & -0.69 \\
100 & 25.01 & 3.84 & 9.61 & -13.11 & 10.98 & -2.78 & 0.73 & -1.47 \\
150 & 15.00 & 5.77 & 4.72 & -17.27 & 14.14 & -3.02 & 1.06 & -1.96 \\
200 & 6.99 & 7.37 & -0.50 & -21.16 & 15.91 & -2.88 & 1.24 & -2.25 \\
250 & 0.23 & 8.61 & -5.50 & -24.86 & 16.77 & -2.58 & 1.21 & -2.45 \\
300 & -5.64 & 9.52 & -10.17 & -28.37 & 17.01 & -2.23 & 0.90 & -2.66 \\
350 & -10.86 & 10.14 & -14.49 & -31.70 & 16.81 & -1.88 & 0.29 & -2.95 \\
\hline \hline
\end{tabular}

starts to increase beyond $\sim 150 \mathrm{MeV}$. We should mention that there are a number of groups of $n p$ total cross section data which extend over a wide energy range. So in order to present the results in the form of Table III, we had to split each of these groups into a number of subgroups, each contributing in its appropriate energy bin. Whenever one of these groups has a normalization error, we choose to apply this same normalization for each of its subgroups. As a consequence, the number of $n p$ data in Table III is increased by 12 , while the total $\chi^{2}(n p)$ is lowered by 28 . The reason for this reduction in $\chi^{2}$ is that these 12 extra normalizations are optimized for each subgroup separately.

As an independent test, we have also checked our results with the scattering analysis interactive dial-in (SAID) program, of the Virginia Polytechnic Institute and State University (VPI\&SU) group [31]. We provided SAID with phase shifts calculated at the 17 energies 1 , $5,10,25(25), 350 \mathrm{MeV}$. The saID program then uses an interpolation routine to provide the phase shifts at all energies at which the experimental data were measured. For the $S$ waves below $25 \mathrm{MeV}$, this interpolation deviates slightly from the actual values of the potential. Moreover, the treatment of the electromagnetic contributions is less sophisticated in the VPI\&SU analysis, which leads to a large discrepancy for laboratory energies below $2 \mathrm{MeV}$. When we then compare with the data between 2 and $350 \mathrm{MeV}$, we obtain a $\chi^{2}(p p)=2107$ for $1644 p p$ data and a $\chi^{2}(n p)=4157$ for $3020 n p$ data, all from their data set NN943. The total $\chi^{2}$ per datum by this comparison is still a very respectable 1.34.

To demonstrate once more the importance of fitting to both $p p$ and $n p$ data, we used the $n p$ version of the Argonne $v_{18}$ potential, included the proper electromagnetic interaction, and confronted it with the $p p$ data. In this way we partially simulate the comparison with the $p p$ data of a potential model fit only to the $n p$ data. Of course, the analogue is not perfect, because in the Argonne $v_{18}$ potential the $l 2, t, l s, l s 2$ parameters in the triplet $T=1$ partial waves are fit to both $p p$ and $n p$ data. Still, this modified $n p$ potential gives a $\chi^{2}$ per datum of 4.4 for the $p p$ data between 2 and $350 \mathrm{MeV}$, which is much worse than the $\chi^{2}$ per datum of 1.1 for the actual $p p$ Argonne $v_{18}$ potential on the same energy interval. Similarly, we can replace the $T=1 n p$ part of the Argonne $v_{18}$ potential by the $T=1 p p$ part and confront it with the $n p$ data. This modified potential then gives a $\chi^{2}$ per datum of 1.8 on the $n p$ data between 2 and 350 $\mathrm{MeV}$, rather than 1.1 for the actual $n p$ Argonne $v_{18}$.

The $L=0$ phase shifts are calculated using the poten-

TABLE V. $n n$ phase shifts in degrees. Energies are in MeV. The ${ }^{1} S_{0}$ includes the full electromagnetic interaction $\left[v^{\mathrm{EM}}(n n)\right]$. The non- $S$ waves only include the nuclear interaction. All phase shifts are with respect to Riccati-Bessel functions.

\begin{tabular}{rcccccccc}
\hline \hline$T_{\text {lab }}$ & \multicolumn{1}{c}{${ }^{1} S_{0}$} & ${ }^{1} D_{2}$ & ${ }^{3} P_{0}$ & ${ }^{3} P_{1}$ & ${ }^{3} P_{2}$ & $\varepsilon_{2}$ & ${ }^{3} F_{2}$ & ${ }^{3} F_{3}$ \\
\hline 1 & 57.07 & 0.00 & 0.21 & -0.12 & 0.02 & -0.00 & 0.00 & -0.00 \\
5 & 60.64 & 0.05 & 1.88 & -1.04 & 0.27 & -0.06 & 0.00 & -0.01 \\
10 & 57.48 & 0.18 & 4.17 & -2.24 & 0.76 & -0.22 & 0.01 & -0.04 \\
25 & 48.80 & 0.74 & 9.13 & -5.12 & 2.69 & -0.86 & 0.11 & -0.24 \\
50 & 38.47 & 1.79 & 11.89 & -8.48 & 6.08 & -1.80 & 0.32 & -0.70 \\
100 & 24.45 & 3.92 & 9.48 & -13.38 & 11.31 & -2.79 & 0.74 & -1.49 \\
150 & 14.38 & 5.87 & 4.46 & -17.58 & 14.45 & -3.00 & 1.07 & -1.98 \\
200 & 6.34 & 7.48 & -0.81 & -21.49 & 16.19 & -2.84 & 1.25 & -2.26 \\
250 & -0.42 & 8.72 & -5.85 & -25.21 & 17.00 & -2.53 & 1.21 & -2.46 \\
300 & -6.31 & 9.62 & -10.54 & -28.73 & 17.20 & -2.18 & 0.88 & -2.68 \\
350 & -11.53 & 10.24 & -14.87 & -32.08 & 16.96 & -1.83 & 0.25 & -2.97 \\
\hline \hline
\end{tabular}


TABLE VI. $n p T=1$ phase shifts in degrees. Energies are in $\mathrm{MeV}$. The ${ }^{1} S_{0}$ includes the full electromagnetic interaction $\left[v^{\mathrm{EM}}(n p)\right]$. The non- $S$ waves only include the nuclear interaction. All phase shifts are with respect to Riccati-Bessel functions.

\begin{tabular}{rcccccccc}
\hline \hline$T_{\text {lab }}$ & \multicolumn{1}{c}{${ }^{1} S_{0}$} & ${ }^{1} D_{2}$ & ${ }^{3} P_{0}$ & ${ }^{3} P_{1}$ & ${ }^{3} P_{2}$ & $\varepsilon_{2}$ & ${ }^{3} F_{2}$ & ${ }^{3} F_{3}$ \\
\hline 1 & 62.02 & 0.00 & 0.18 & -0.11 & 0.02 & -0.00 & 0.00 & -0.00 \\
5 & 63.50 & 0.04 & 1.64 & -0.93 & 0.26 & -0.05 & 0.00 & -0.00 \\
10 & 59.78 & 0.16 & 3.71 & -2.04 & 0.72 & -0.19 & 0.01 & -0.03 \\
25 & 50.61 & 0.68 & 8.32 & -4.82 & 2.57 & -0.77 & 0.08 & -0.20 \\
50 & 40.09 & 1.70 & 10.99 & -8.15 & 5.86 & -1.68 & 0.28 & -0.61 \\
100 & 26.02 & 3.81 & 8.69 & -13.07 & 11.00 & -2.69 & 0.67 & -1.35 \\
150 & 15.98 & 5.72 & 3.78 & -17.28 & 14.12 & -2.95 & 0.98 & -1.82 \\
200 & 8.00 & 7.30 & -1.43 & -21.22 & 15.86 & -2.82 & 1.15 & -2.10 \\
250 & 1.28 & 8.52 & -6.41 & -24.95 & 16.70 & -2.54 & 1.10 & -2.30 \\
300 & -4.54 & 9.43 & -11.06 & -28.49 & 16.91 & -2.21 & 0.77 & -2.51 \\
350 & -9.71 & 10.06 & -15.36 & -31.85 & 16.69 & -1.88 & 0.14 & -2.81 \\
\hline \hline
\end{tabular}

tials discussed in Sec. II, i.e., including the complete electromagnetic interaction, and by matching to electromagnetic wave functions $\left(\delta_{\mathrm{EM}+N}^{\mathrm{EM}}\right.$ in the notation of Ref. [32]). For $L \neq 0$ we use the fact that $\delta_{\mathrm{EM}+N}^{\mathrm{EM}}$ can be reasonably approximated $[32,16]$ by only including the Coulomb interaction with $\alpha^{\prime}$ (in case of $p p$ scattering) or no electromagnetic interaction at all (in the case of $n p$ or $n n$ scattering). The resulting phase shifts for partial waves with $J \leq 3$ are shown in Table IV for $p p$ scattering, in Table $\mathrm{V}$ for $n n$, in Table VI for $n p$ in $T=1$ states, and in Table VII for $n p$ in $T=0$ states. Note that the non- $S$ $p p$ phase shifts in Table IV are calculated including the form factor $F_{C}(r)$ in the Coulomb potential $V_{C 1}(p p)$.

In addition, we show figures of some of the more interesting phases and compare to the Nijmegen multienergy partial-wave analysis [16], the single-energy analysis from SAID [31], and recent single-energy analyses by Bugg and Bryan [33], and by Henneck [34]. In Fig. 2 the $p p, n n$, and $n{ }^{1} S_{0}$ phases of Argonne $v_{18}$ are shown, and seen to be in good agreement with the various analyses. The charge dependence is clearly evident; a discussion of the relative size of various contributions to charge-independence breaking is given below in Sec. IV. The ${ }^{3} P_{0}$ phases are shown in Fig. 3 ; the ${ }^{3} P_{0}$ channel displays the second greatest amount of CIB after the ${ }^{1} S_{0}$ channel. Again there seems to be reasonable agreement with the various partial-wave analyses. The $\epsilon_{1}$ mixing parameter, shown in Fig. 4, is both the most difficult to determine in singleenergy analyses, as indicated by the range of values and size of error bars, and one of the most important because of its relation to the strength of the tensor interaction. The Argonne $v_{18}$ value tracks the Nijmegen multienergy analysis up to $T_{\text {lab }}=100 \mathrm{MeV}$ before deviating slightly on the high side. However, the differences with the Nijmegen multienergy analysis are still within two standard deviations. Finally, the ${ }^{1} P_{1}$ phase shift, which is intimately related to the $\epsilon_{1}$ mixing parameter, is shown in Fig. 5. Here the present model is somewhat less repulsive than the various partial-wave analyses above 150 $\mathrm{MeV}$.

The low-energy scattering parameters are shown in Table VIII and compared to experimental results $[27,32,35]$. The scattering lengths and effective ranges are calculated both with and without the electromagnetic interaction. Without the electromagnetic interaction, the effective range function is simply given by $F\left(k^{2}\right)=k \cot \delta_{N}=$ $-1 / a+\frac{1}{2} r k^{2}+\mathcal{O}\left(k^{4}\right)$. In the presence of the electromagnetic interaction, we have to use a more complicated effective range function [32], where the phase shifts are with respect to the full long-range electromagnetic interaction.

TABLE VII. $n p T=0$ phase shifts in degrees. Energies are in $\mathrm{MeV}$. The coupled ${ }^{3} S_{1-}{ }^{3} D_{1}$ channel includes the full electromagnetic interaction $\left[v^{\mathrm{EM}}(n p)\right]$. The non- $S$ waves only include the nuclear interaction. All phase shifts are with respect to Riccati-Bessel functions.

\begin{tabular}{rccrcccccc}
\hline \hline$T_{\text {lab }}$ & \multicolumn{1}{c}{${ }^{1} P_{1}$} & \multicolumn{1}{c}{${ }^{1} F_{3}$} & \multicolumn{1}{c}{${ }^{3} S_{1}$} & $\varepsilon_{1}$ & ${ }^{3} D_{1}$ & ${ }^{3} D_{2}$ & ${ }^{3} D_{3}$ & $\varepsilon_{3}$ & ${ }^{3} G_{3}$ \\
\hline 1 & -0.19 & -0.00 & 147.75 & 0.11 & -0.00 & 0.01 & 0.00 & 0.00 & -0.00 \\
5 & -1.51 & -0.01 & 118.18 & 0.66 & -0.17 & 0.22 & 0.00 & 0.01 & -0.00 \\
10 & -3.11 & -0.07 & 102.62 & 1.14 & -0.65 & 0.85 & 0.01 & 0.08 & -0.00 \\
25 & -6.48 & -0.42 & 80.68 & 1.77 & -2.72 & 3.71 & 0.08 & 0.55 & -0.05 \\
50 & -9.85 & -1.13 & 62.89 & 2.11 & -6.28 & 8.94 & 0.40 & 1.61 & -0.26 \\
100 & -14.20 & -2.22 & 43.51 & 2.52 & -12.04 & 17.10 & 1.61 & 3.50 & -0.93 \\
150 & -17.68 & -2.98 & 31.19 & 2.96 & -16.39 & 21.85 & 2.92 & 4.88 & -1.74 \\
200 & -20.79 & -3.61 & 21.94 & 3.43 & -19.82 & 24.20 & 4.00 & 5.88 & -2.58 \\
250 & -23.65 & -4.22 & 14.45 & 3.92 & -22.59 & 25.06 & 4.76 & 6.61 & -3.41 \\
300 & -26.28 & -4.87 & 8.13 & 4.43 & -24.83 & 25.01 & 5.21 & 7.16 & -4.20 \\
350 & -28.71 & -5.59 & 2.65 & 4.95 & -26.65 & 24.41 & 5.39 & 7.59 & -4.96 \\
\hline \hline
\end{tabular}



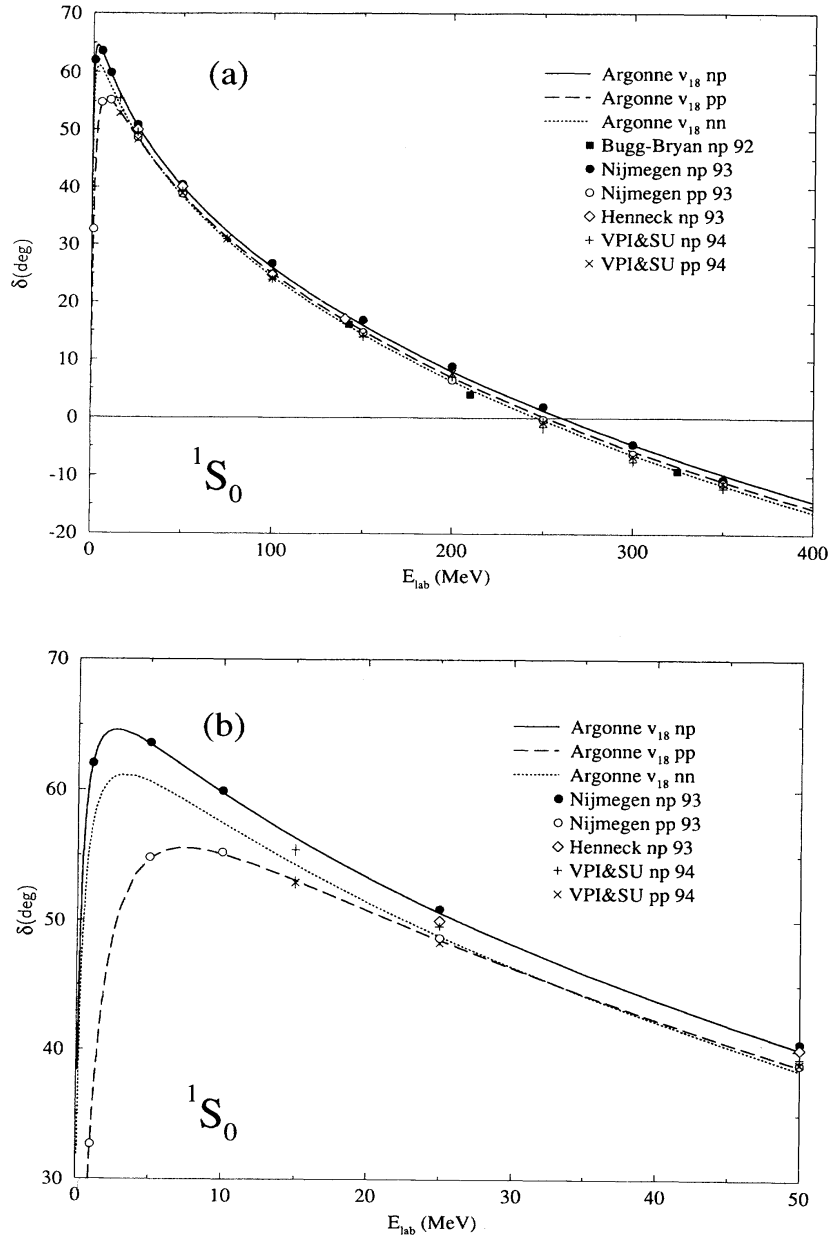

FIG. 2. Phase shifts in the ${ }^{1} S_{0}$ channel for $n p, n n$, and $p p$ scattering, compared to various partial-wave phase-shift analyses.

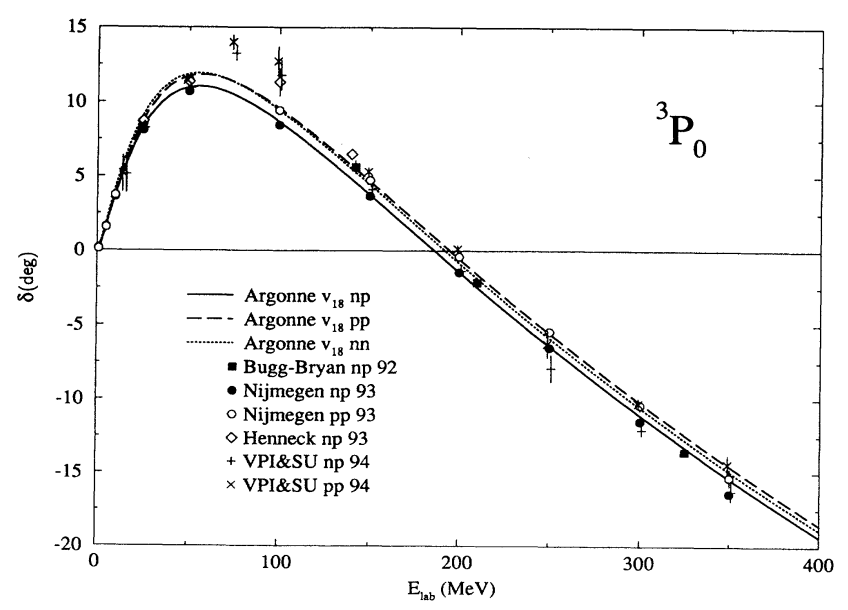

FIG. 3. Phase shifts in the ${ }^{3} P_{0}$ channel for $n p, n n$, and $p p$ scattering, compared to various partial-wave phase-shift analyses.

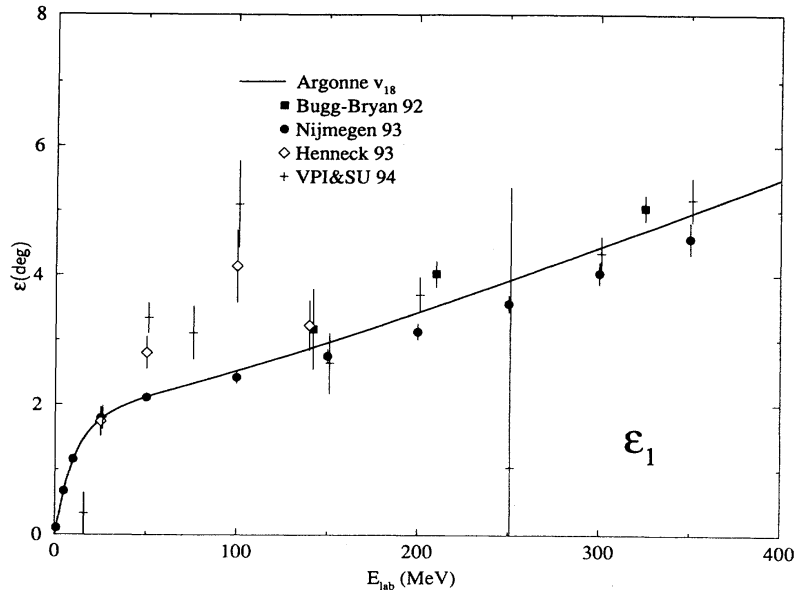

FIG. 4. The $\epsilon_{1}$ mixing parameter compared to various partial-wave phase-shift analyses.

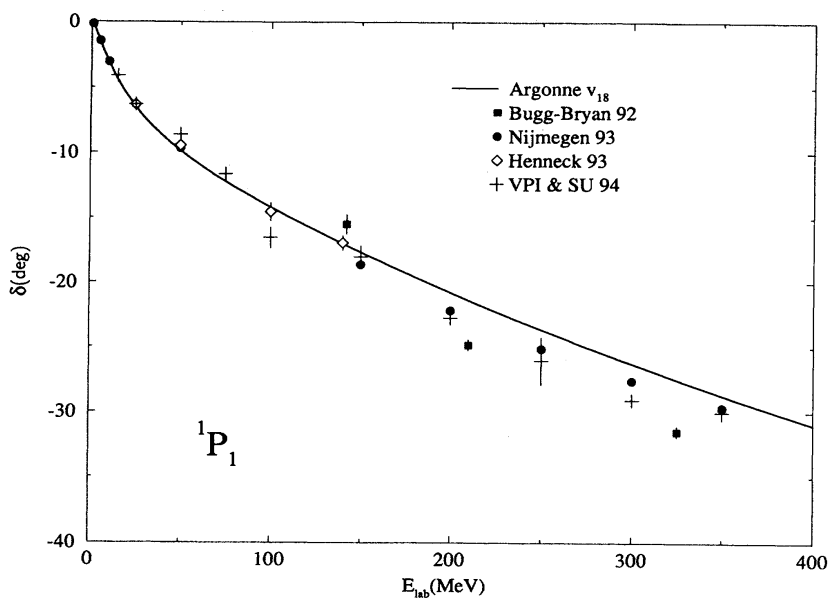

FIG. 5. Phase shifts in the ${ }^{1} P_{1}$ channel, compared to various partial-wave phase-shift analyses.

TABLE VIII. Scattering lengths and effective ranges in $\mathrm{fm}$.

\begin{tabular}{lccr}
\hline & Experiment & Argonne $v_{18}$ & \multicolumn{1}{c}{ w/o $v^{\mathrm{EM}}$} \\
\hline${ }^{1} a_{p p}$ & $-7.8063 \pm 0.0026^{\mathrm{a}}$ & -7.8064 & -17.164 \\
${ }^{1} r_{p p}$ & $2.794 \pm 0.014^{\mathrm{a}}$ & 2.788 & 2.865 \\
${ }^{1} a_{n n}$ & $-18.5 \pm 0.4^{\mathrm{b}}$ & -18.487 & -18.818 \\
${ }^{1} r_{n n}$ & $2.80 \pm 0.11^{\mathrm{b}}$ & 2.840 & 2.834 \\
${ }^{1} a_{n p}$ & $-23.749 \pm 0.008^{\mathrm{c}}$ & -23.732 & -23.084 \\
${ }^{1} r_{n p}$ & $2.81 \pm 0.05^{\mathrm{c}}$ & 2.697 & 2.703 \\
${ }^{3} a_{n p}$ & $5.424 \pm 0.003^{\mathrm{c}}$ & 5.419 & 5.402 \\
${ }^{3} r_{n p}$ & $1.760 \pm 0.005^{\mathrm{c}}$ & 1.753 & 1.752 \\
\hline \hline
\end{tabular}

${ }^{\mathrm{a}}$ Reference [32].

${ }^{\mathrm{b}}$ Reference [28].

${ }^{\mathrm{c}}$ Reference [35]. 


\section{PROJECTION INTO OPERATOR FORMAT}

We can project the strong interaction potential given above from $S, T, T_{z}$ states into an operator format with 18 terms

$$
v_{i j}=\sum_{p=1,18} v_{p}\left(r_{i j}\right) O_{i j}^{p} .
$$

Here the first 14 operators are the same chargeindependent ones used in the Argonne $v_{14}$ potential and are given by

$$
\begin{aligned}
O_{i j}^{p=1,14}= & 1, \boldsymbol{\tau}_{i} \cdot \boldsymbol{\tau}_{j}, \boldsymbol{\sigma}_{i} \cdot \boldsymbol{\sigma}_{j},\left(\boldsymbol{\sigma}_{i} \cdot \boldsymbol{\sigma}_{j}\right)\left(\boldsymbol{\tau}_{i} \cdot \boldsymbol{\tau}_{j}\right), S_{i j}, S_{i j}\left(\boldsymbol{\tau}_{i} \cdot \boldsymbol{\tau}_{j}\right), \mathbf{L} \cdot \mathbf{S}, \mathbf{L} \cdot \mathbf{S}\left(\boldsymbol{\tau}_{i} \cdot \boldsymbol{\tau}_{j}\right) \\
& L^{2}, L^{2}\left(\boldsymbol{\tau}_{i} \cdot \boldsymbol{\tau}_{j}\right), L^{2}\left(\boldsymbol{\sigma}_{i} \cdot \boldsymbol{\sigma}_{j}\right), L^{2}\left(\boldsymbol{\sigma}_{i} \cdot \boldsymbol{\sigma}_{j}\right)\left(\boldsymbol{\tau}_{i} \cdot \boldsymbol{\tau}_{j}\right),(\mathbf{L} \cdot \mathbf{S})^{2},(\mathbf{L} \cdot \mathbf{S})^{2}\left(\boldsymbol{\tau}_{i} \cdot \boldsymbol{\tau}_{j}\right)
\end{aligned}
$$

These 14 components are denoted by the abbreviations $c, \tau, \sigma, \sigma \tau, t, t \tau, l s, l s \tau, l 2, l 2 \tau, l 2 \sigma, l 2 \sigma \tau, l s 2$, and $l s 2 \tau$. The four additional operators break charge independence and are given by

$$
O_{i j}^{p=15,18}=T_{i j},\left(\sigma_{i} \cdot \sigma_{j}\right) T_{i j}, S_{i j} T_{i j},\left(\tau_{z i}+\tau_{z j}\right),
$$

where $T_{i j}=3 \tau_{z i} \tau_{z j}-\tau_{i} \cdot \tau_{j}$ is the isotensor operator, defined analogous to the $S_{i j}$ operator. These terms are abbreviated as $T, \sigma T, t T$, and $\tau z$. The $T, \sigma T$, and $t T$ operators are charge dependent and are "class II" forces, while the $\tau z$ operator is charge asymmetric and is a "class III" force [25].

The operator potential terms, $v_{p}$, can be obtained from the channel potentials, $v_{S T, N N}^{x}$, by a simple set of projections. We first introduce charge splitting for the central $T=1$ states,

$$
v_{S 1, N N}^{c}=v_{S 1}^{\mathrm{CI}}+v_{S 1}^{\mathrm{CD}} T_{i j}+v_{S 1}^{\mathrm{CA}}\left(\tau_{z i}+\tau_{z j}\right) .
$$

For the charge-independent potential this implies

$$
v_{S 1}^{\mathrm{CI}}=\frac{1}{3}\left(v_{S 1, p p}^{c}+v_{S 1, n n}^{c}+v_{S 1, n p}^{c}\right) \text {. }
$$

We then project

$$
\begin{aligned}
v_{c} & =\frac{1}{16}\left(9 v_{11}^{\mathrm{CI}}+3 v_{10}^{\mathrm{CI}}+3 v_{01}^{\mathrm{CI}}+v_{00}^{\mathrm{CI}}\right), \\
v_{\tau} & =\frac{1}{16}\left(3 v_{11}^{\mathrm{CI}}-3 v_{10}^{\mathrm{CI}}+v_{01}^{\mathrm{CI}}-v_{00}^{\mathrm{CI}}\right), \\
v_{\sigma} & =\frac{1}{16}\left(3 v_{11}^{\mathrm{CI}}+v_{10}^{\mathrm{CI}}-3 v_{01}^{\mathrm{CI}}-v_{00}^{\mathrm{CI}}\right), \\
v_{\sigma \tau} & =\frac{1}{16}\left(v_{11}^{\mathrm{CI}}-v_{10}^{\mathrm{CI}}-v_{01}^{\mathrm{CI}}+v_{00}^{\mathrm{CI}}\right),
\end{aligned}
$$

where of course $v_{10}^{\mathrm{CI}}=v_{10, n p}^{c}$ and $v_{00}^{\mathrm{CI}}=v_{00, n p}^{c}$. A similar set of projections is used for the $L^{2}$ parts of the interaction. For the tensor, spin-orbit, and quadratic spin-orbit pieces, which exist only in $S=1$ channels, the projections are $(x=t, l s, l s 2)$

$$
\begin{aligned}
v_{x} & =\frac{1}{4}\left(3 v_{11}^{x}+v_{10}^{x}\right), \\
v_{x \tau} & =\frac{1}{4}\left(v_{11}^{x}-v_{10}^{x}\right) .
\end{aligned}
$$

The charge-dependent terms in Eq. (28) are given by

$$
v_{S 1}^{\mathrm{CD}}=\frac{1}{6}\left[\frac{1}{2}\left(v_{S 1, p p}^{c}+v_{S 1, n n}^{c}\right)-v_{S 1, n p}^{c}\right],
$$

which can be projected as

$$
\begin{aligned}
v_{T} & =\frac{1}{4}\left(3 v_{11}^{\mathrm{CD}}+v_{01}^{\mathrm{CD}}\right), \\
v_{\sigma T} & =\frac{1}{4}\left(v_{11}^{\mathrm{CD}}-v_{01}^{\mathrm{CD}}\right) .
\end{aligned}
$$

The charge-dependent tensor term comes only from the spin-triplet channel, and reads

$$
v_{t T}=\frac{1}{6}\left[\frac{1}{2}\left(v_{11, p p}^{t}+v_{11, n n}^{t}\right)-v_{11, n p}^{t}\right] .
$$

Finally, the charge-asymmetric terms are given by

$$
v_{S 1}^{\mathrm{CA}}=\frac{1}{4}\left(v_{S 1, p p}^{c}-v_{S 1, n n}^{c}\right)
$$

which leads to

$$
\begin{aligned}
v_{\tau z} & =\frac{1}{4}\left(3 v_{11}^{\mathrm{CA}}+v_{01}^{\mathrm{CA}}\right), \\
v_{\sigma \tau z} & =\frac{1}{4}\left(v_{11}^{\mathrm{CA}}-v_{01}^{\mathrm{CA}}\right) .
\end{aligned}
$$

As discussed in the previous section, we fix $v_{01}^{\mathrm{CA}}$ to reproduce the singlet $n n$ scattering length by adjusting the parameter $P_{01, n n}^{c}$ to be slightly different from $P_{01, p p}^{c}$. We are unaware of any $n n$ data that would allow us to fix $v_{11}^{\mathrm{CA}}$, but there have been numerous theoretical predictions for charge-symmetry breaking based on $\rho-\omega$ and $\pi-\eta-\eta^{\prime}$ mixing. Such models suggest that $v_{11}^{\mathrm{CA}}$ should be somewhat larger than $v_{01}^{\mathrm{CA}}$, but with a similar shape [36]. In the present work we make the simple assumption $v_{11}^{\mathrm{CA}}=v_{01}^{\mathrm{CA}}$, which implies there is no $v_{\sigma \tau z}$ term. We also neglect the possibility of a charge-asymmetric tensor term $v_{t \tau z}$, which is why we end up with only one charge-asymmetric

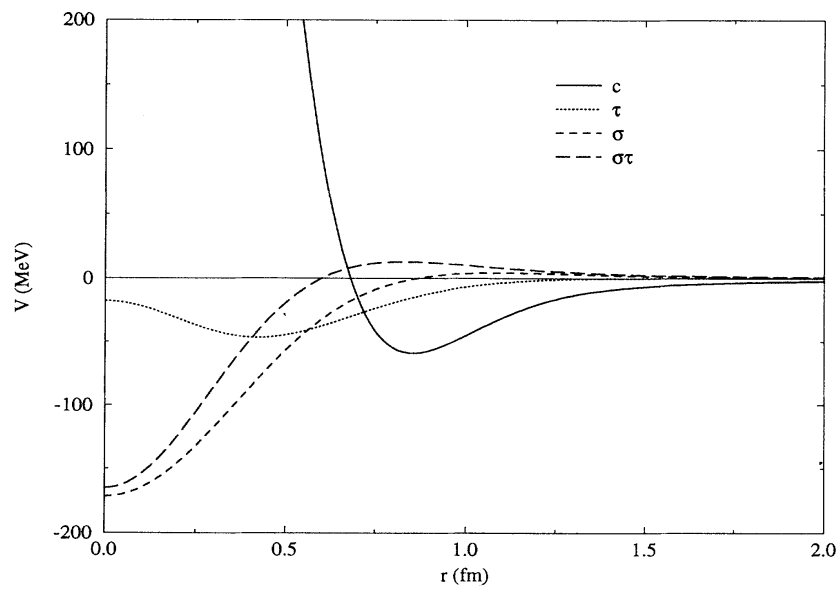

FIG. 6. Central, isospin, spin, and spin-isospin components of the potential. The central potential has a peak value of $2031 \mathrm{MeV}$ at $r=0$. 


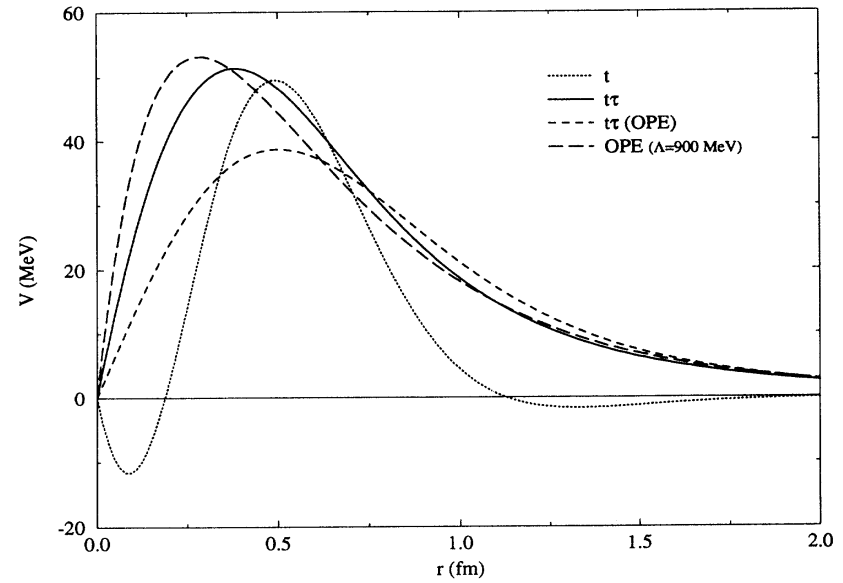

FIG. 7. Tensor and tensor-isospin parts of the potential. Also shown are the OPE contribution to the tensor-isospin potential, and for comparison an OPE potential with a monopole form factor containing a $900 \mathrm{MeV}$ cutoff mass.

operator in our model. These choices are reflected in the parameters of Table II.

The first four operator components of the potential are shown in Fig. 6. The tensor components are shown in Fig. 7 where we also show the CI part of the OPE potential used here, and for comparison an OPE potential constructed using the same coupling constant and a dipole form factor (monopole at each nucleon-nucleon-pion vertex) with the cutoff mass $\Lambda=900 \mathrm{MeV}$. The spin-orbit and quadratic spin-orbit terms are shown in Fig. 8, while the various $L^{2}$ components are shown in Fig. 9. Finally, the charge-dependent and charge-asymmetric terms are shown in Fig. 10, along with the static Coulomb potential for comparison.

The relative importance of the different CIB components is illustrated in Table IX, where the evolution from the CI part of the interaction to the full $p p$ interaction

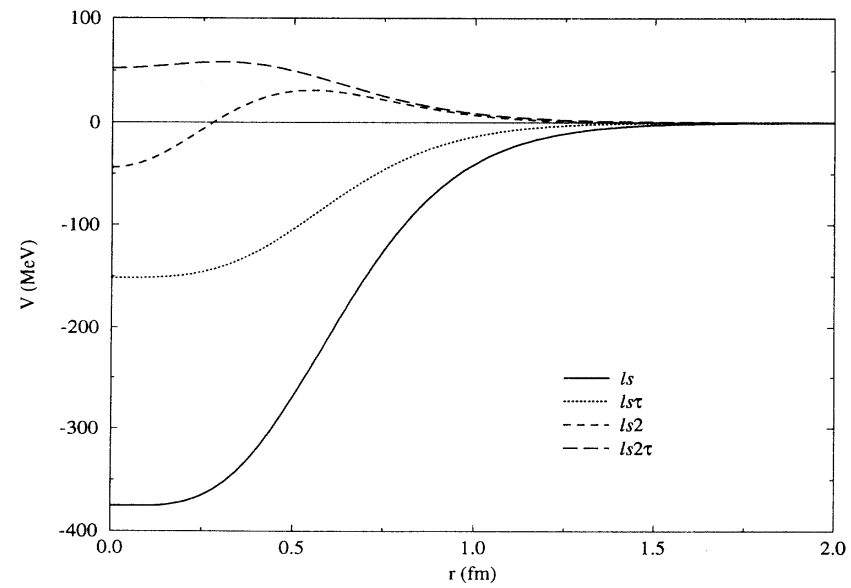

FIG. 8. Spin-orbit and quadratic spin-orbit components of the potential.

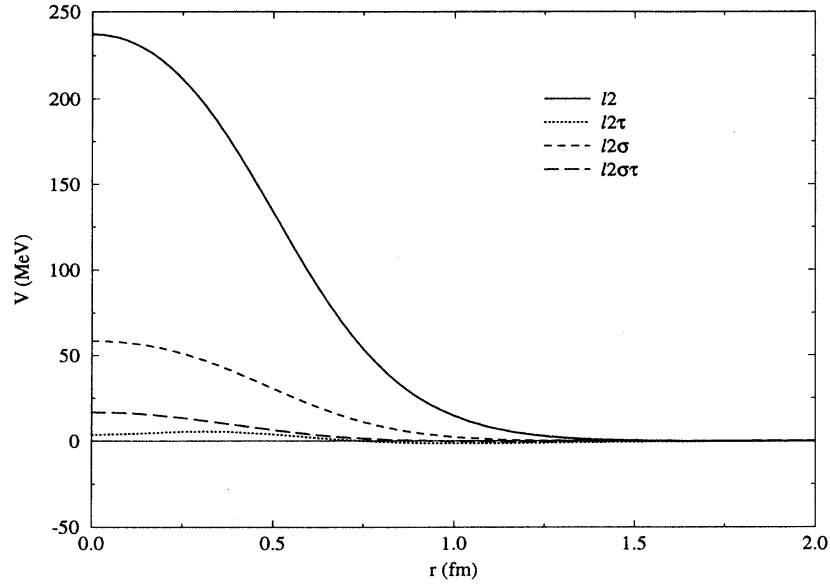

FIG. 9. $L^{2}$ components of the potential.

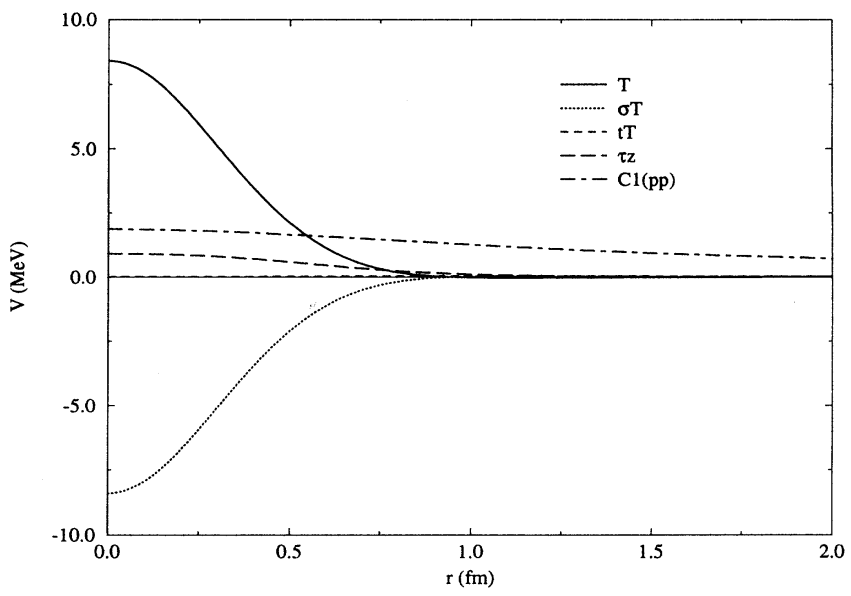

FIG. 10. Charge-dependent and charge-asymmetric components of the potential. Also shown for comparison is the Coulomb potential, $V_{C 1}(p p)$.

TABLE IX. Evolution of ${ }^{1} S_{0} p p$ phase shifts from the charge-independent potential to the full interaction, as described in the text. Energies are in $\mathrm{MeV}$.

\begin{tabular}{rrrrrr}
\hline \hline$T_{\text {lab }}$ & \multicolumn{1}{c}{ CI } & $+m_{p}$ & + CD $v^{\pi}$ & + CD $v^{R}$ & $+v^{\mathrm{EM}}$ \\
\hline 1 & 57.99 & 57.80 & 57.42 & 55.50 & 32.68 \\
5 & 61.22 & 61.12 & 60.88 & 59.78 & 54.74 \\
10 & 57.98 & 57.90 & 57.71 & 56.84 & 55.09 \\
25 & 49.22 & 49.17 & 49.05 & 48.36 & 48.51 \\
50 & 38.87 & 38.84 & 38.76 & 38.13 & 38.78 \\
100 & 24.87 & 24.85 & 24.80 & 24.19 & 25.01 \\
150 & 14.83 & 14.81 & 14.77 & 14.16 & 15.00 \\
200 & 6.82 & 6.80 & 6.77 & 6.15 & 6.99 \\
250 & 0.08 & 0.06 & 0.04 & -0.60 & 0.23 \\
300 & -5.78 & -5.79 & -5.82 & -6.47 & -5.64 \\
350 & -10.99 & -11.00 & -11.01 & -11.69 & -10.86 \\
\hline \hline
\end{tabular}


TABLE X. Static deuteron properties.

\begin{tabular}{|c|c|c|c|c|}
\hline & Experiment & Argonne $v_{18}$ & $+\mathrm{R}+\mathrm{MEC}$ & Units \\
\hline$E_{d}$ & $2.224575(9)^{\mathrm{a}}$ & 2.224575 & & $\mathrm{MeV}$ \\
\hline$\langle T\rangle$ & & 19.814 & & $\mathrm{MeV}$ \\
\hline$\left\langle v^{\mathrm{EM}}\right\rangle$ & & 0.018 & & $\mathrm{MeV}$ \\
\hline$\left\langle v^{\pi}\right\rangle^{\prime}$ & & -21.286 & & $\mathrm{MeV}$ \\
\hline$\left\langle v^{R}\right\rangle$ & & -0.770 & & $\mathrm{MeV}$ \\
\hline$A_{S}$ & $0.8781(44)^{b}$ & 0.8850 & & $\mathrm{fm}^{1 / 2}$ \\
\hline$\eta$ & $0.0256(4)^{c}$ & 0.0250 & & \\
\hline$r_{d}$ & $1.953(3)^{\mathrm{d}}$ & 1.967 & & $\mathrm{fm}$ \\
\hline$\mu_{d}$ & $0.857406(1)^{\mathrm{e}}$ & 0.847 & 0.871 & $\mu_{0}$ \\
\hline$Q_{d}$ & $0.2859(3)^{f}$ & 0.270 & 0.275 & $\mathrm{fm}^{2}$ \\
\hline$P_{d}$ & & 5.76 & & $\%$ \\
\hline $\begin{array}{l}\text { a Reference [37]. } \\
\text { b Reference [38]. } \\
\text { c Reference [39]. }\end{array}$ & & $\begin{array}{l}{ }^{\mathrm{d}} \text { Reference [ } \\
{ }^{\mathbf{e}} \text { Reference [ } \\
\text { f Reference [ }\end{array}$ & & \\
\hline
\end{tabular}

is displayed. The successive columns give the ${ }^{1} S_{0}$ phase shifts for (1) the CI potential with an average nucleon and average pion mass, (2) with the correct proton mass, (3) with the correct CD OPE tail (i.e., correct neutralpion mass) but the CI core, (4) with both the CD OPE and core interactions, and (5) with the electromagnetic potential added. From these it can be seen that the nucleon mass has a relatively small effect, while the CD OPE and core terms have relatively large effects at low energy, and the core contribution becomes dominant at higher energies.

\section{DEUTERON PROPERTIES}

The static deuteron properties are shown in Table $\mathrm{X}$ and compared to experimental values [37-42]. The binding energy, $E_{d}$, is fit exactly by construction. The expectation values for the kinetic energy, $T$, and for the EM, OPE, and remaining potentials are also shown. We note that the OPE potential dominates, while the EM potential gives a small but non-negligible $18 \mathrm{keV}$ contribution, mostly from the magnetic moment term. The asymptotic $S$-state normalization, $A_{S}$, and the $D / S$ ratio, $\eta$, are both $\sim 1.5$ standard deviations from experiment. The deuteron radius, $r_{d}$, is off rather more, which is a persistent problem with $N N$ potential models. The magnetic moment, $\mu_{d}$, and the quadrupole moment, $Q_{d}$, are both underpredicted in impulse approximation; both have significant relativistic and meson-exchange corrections, as discussed below. (Such corrections to $r_{d}$ are quite small.) Finally, the $D$-state percentage is about $5 \%$ smaller than that of the older Argonne $v_{14}$ model [1] and almost identical to that of the Paris potential [7].

The $S$ - and $D$-wave components of the deuteron wave function are shown in Fig. 11, where they are compare to those for the older $v_{14}$ model. The short-range behavior of the wave function components is moderately different. The $A\left(q^{2}\right)$ and $B\left(q^{2}\right)$ structure functions and tensor polarization $T_{20}\left(q^{2}\right)$ obtained with the present interaction model are displayed in Figs. 12-14; the experimental data are from Refs. [24,43-51]. The model for the isoscalar electromagnetic current operator has been discussed in detail in Refs. [52,53], here we only summarize its general structure, which consists of one- and twobody parts. The one-body part has the standard impulse approximation (IA) form, with inclusion, in the charge component, of the Darwin-Foldy and spin-orbit relativistic corrections [54]. The two-body charge operators contain contributions that correspond (in an OBE picture) to those obtained from pion- and vector-meson ( $\rho$ and $\omega)$ exchanges. These are obtained from the nonrelativistic reduction of the Born terms in the corresponding relativistic photoproduction amplitudes [54]. The two-body current operators are constructed from the spin-orbit and quadratic momentum-dependent components of the interaction with the methods developed in Refs. [52,55]. We also consider the two-body charge and current operators associated with the $\rho \pi \gamma$ mechanism. In particular, we include in the nonrelativistic reduction of its current component the next to leading order correction arising from the tensor coupling of the $\rho$ meson to the nucleon [53]. The Höhler parametrization 8.2 [56] is used for the electromagnetic form factors of the nucleon, while an $\omega$-pole term form factor is included at the $\rho \pi \gamma$ electromagnetic vertex.

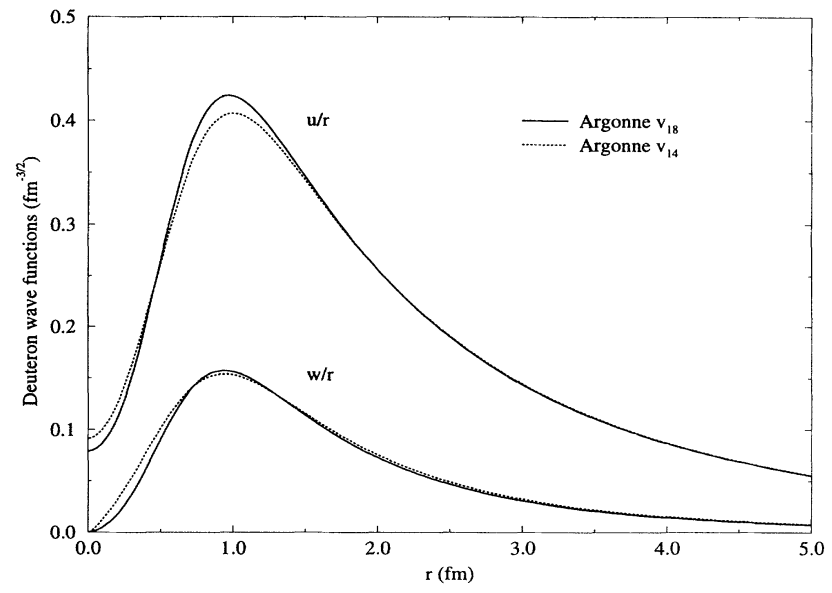

FIG. 11. The deuteron $S$ - and $D$-wave function components divided by $r$. 


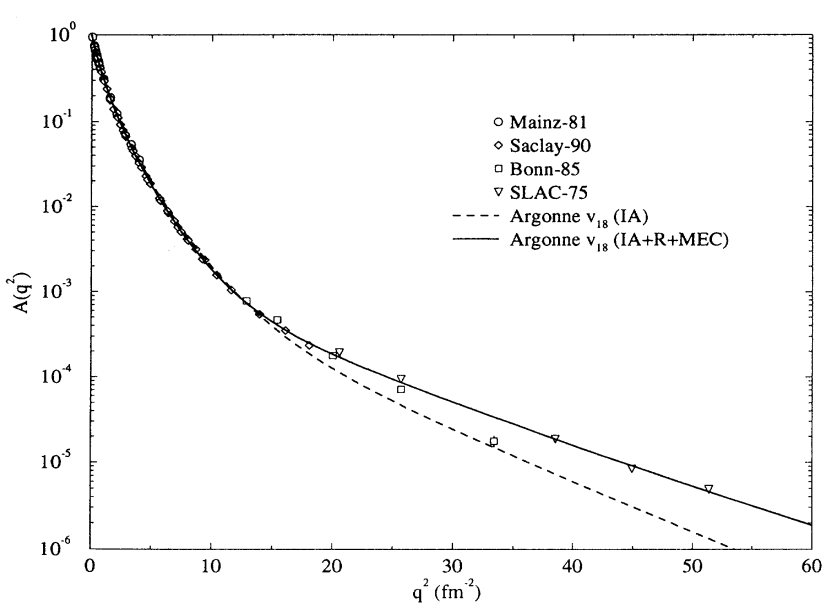

FIG. 12. The deuteron electromagnetic structure function $A\left(q^{2}\right)$ in impulse approximation (dashed line) and with relativistic and exchange-current corrections (solid line). Data are from Bonn [44], Mainz [43], Saclay [24], and SLAC [45].

The calculated $A\left(q^{2}\right)$ structure function is in excellent agreement with the experimental data over the whole range of measured momentum transfers. The DarwinFoldy and spin-orbit relativistic corrections to the singlenucleon charge operator as well as the leading two-body charge contribution due to pion exchange play an important role, as it is evident from Fig. 12. However, these same contributions lead to a significant discrepancy between theory and experiment in the tensor polarization. This observable and the $A\left(q^{2}\right)$ structure function are mostly sensitive to the charge and quadrupole form factors. In particular, the momentum transfer at which the minimum of $T_{20}\left(q^{2}\right)$ occurs is related to the position of the charge form factor zero. The relative shift between the predicted and experimental $T_{20}\left(q^{2}\right)$ minima implies, therefore, a corresponding shift between the charge form factor zeros.

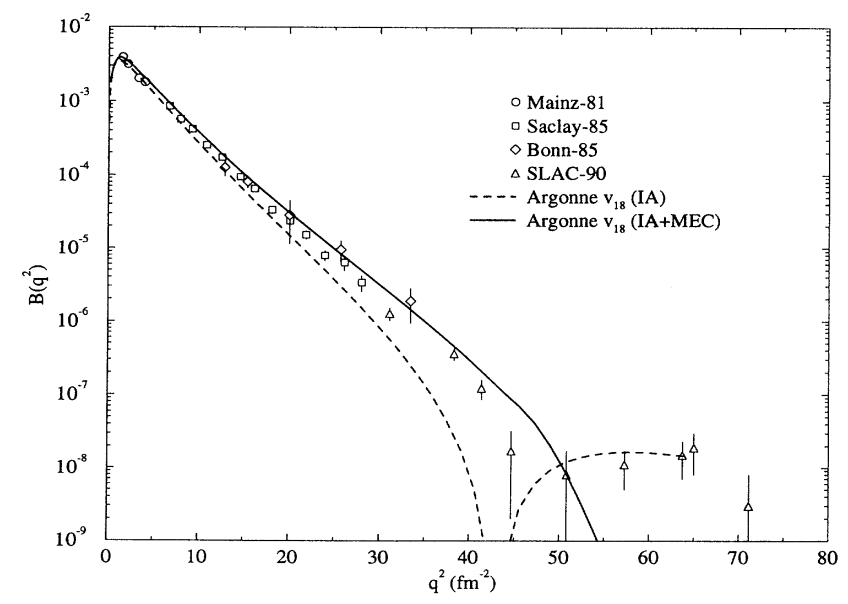

FIG. 13. The deuteron electromagnetic structure function $B\left(q^{2}\right)$ in impulse approximation (dashed line) and with exchange-current corrections (solid line). Data are from Bonn [44], Mainz [43], Saclay [46], and SLAC [47].

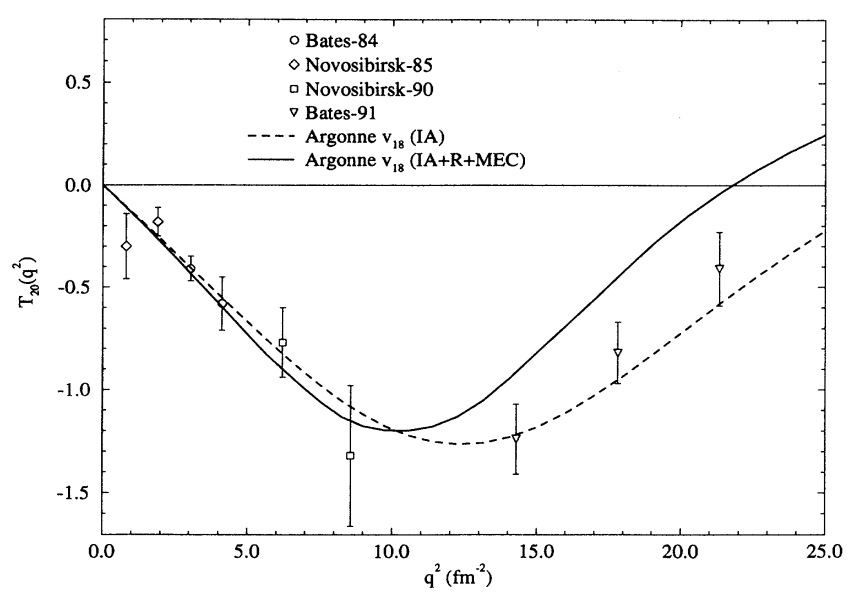

FIG. 14. The deuteron tensor polarization $T_{20}$ in impulse approximation (dashed line) and with relativistic and exchange-current corrections (solid line). Data are from Bates $[48,49]$ and Novosibirsk $[50,51]$.

The calculated $B\left(q^{2}\right)$ structure function is found to overpredict the experimental data in the momentum transfer range $10-45 \mathrm{fm}^{-2}$, and has a zero around 60 $\mathrm{fm}^{-2}$. The leading two-body contributions are those due to the spin-orbit and quadratic spin-orbit components of the interaction. They are of opposite sign. However, the overestimate of the data indicates that the degree of cancellation between them is not quite enough. The $\rho \pi \gamma$ current contribution is small over the momentum transfer range considered here (we have used the rather soft cutoff values of $0.75 \mathrm{GeV}$ and $1.25 \mathrm{GeV}$ at the $\pi N N$ and $\rho N N$ vertices, respectively, as suggested in Ref. [57]). At present, the two-body currents associated with the quadratic spin-orbit and $L^{2}$ components of the interaction are essentially obtained by minimal substitution $\mathbf{p}_{i} \rightarrow \mathbf{p}_{i}-\left[G_{E}^{s}\left(q^{2}\right)+G_{E}^{v}\left(q^{2}\right) \tau_{z, i}\right] \mathbf{A}\left(\mathbf{r}_{i}\right)$, where $\mathbf{A}$ is the vector potential, $G_{E}^{s}$ and $G_{E}^{v}$ the isoscalar and isovector nucleon electric form factors [52]. It would be desirable to construct these current components in a more systematic way, as suggested in Ref. [58].

Finally, the values for the quadrupole and magnetic moments obtained with the full charge and current operators are $Q_{d}=0.275 \mathrm{fm}^{2}$ and $\mu_{d}=0.871 \mu_{0}$. The measured quadrupole moment is underestimated by roughly $4 \%$, while the measured magnetic moment is overestimated by $1.5 \%$. The two-body charge and current contributions amount to $2 \%$ and $3 \%$ increases of the IA values for $Q_{d}$ and $\mu_{d}$, as listed in Table X.

\section{CONCLUSIONS AND OUTLOOK}

We have constructed a nonrelativistic $N N$ potential with a local operator structure that gives an excellent fit to $p p$ and $n p$ scattering data, as well as to low-energy $n n$ scattering and the deuteron binding energy. We have projected the potential into charge-independent, chargedependent, and charge-asymmetric pieces. In $T=0$ many-body systems only the CI part of the potential will 
contribute, while the CA part will contribute in systems with $T \geq \frac{1}{2}$ and the CD part for $T \geq 1$ systems. Because of the isotensor projection, the CI part automatically has the correct average of $\frac{2}{3} p p$ (or $n n$ ) and $\frac{1}{3} n p T=1$ interaction in the trinucleons, thus serving as a correct reference point for building $N N N$ potentials. The CA part will contribute to the energy differences of mirror nuclei (the Nolen-Schiffer anomaly [10]), while the CD part will contribute to the splitting of isobaric analog states, e.g., in the $A=6$ nuclei. Studies of these effects are in progress.

We have also computed the deuteron electromagnetic properties in both impulse approximation and with relativistic and exchange-current corrections. The least satisfactory prediction of the potential is the small value for $Q_{d}$, even after corrections are added. The full $4 \%$ discrepancy between the predicted and empirical $Q_{d}$ values is unlikely to be resolved by additional relativistic and/or two-body corrections not included in the present calculation. Similar low values were found by the Nijmegen group in their recent fits [14], which used rather different potential forms than the present model. We are less concerned about the $1.5 \%$ error in the magnetic moment or the overprediction of the $B\left(q^{2}\right)$ structure function because of the uncertainties in the exchange currents discussed above. The $A\left(q^{2}\right)$ structure function is very well reproduced, while the experimental tensor polarization $T_{20}\left(q^{2}\right)$ still has rather large error bars above $10 \mathrm{fm}^{-2}$

Compared to the older Argonne $v_{14}$ potential, the present model has a weaker tensor force, which will generally lead to more binding in light nuclei and less rapid saturation in nuclear matter. This is counteracted by the weaker attraction in $T=1 N N$ states because of the mix of $p p$ and $n p$ components. Initial calculations of few-body nuclei with the $v_{18}$ model show a slight net reduction in the binding energies of ${ }^{3} \mathrm{H}$ and ${ }^{4} \mathrm{He}$ compared to the $v_{14}$ model. Another feature of the new model is a moderately greater attraction in $P$ waves. Few-body nuclei are not sensitive to this part of the $N N$ interaction. However, preliminary calculations [59] of the binding energy of ${ }^{16} \mathrm{O}$ show a significant improvement in the relative stability of ${ }^{16} \mathrm{O}$ and ${ }^{4} \mathrm{He}$, which has been a persistent problem [12]. We believe the Argonne $v_{18}$ potential has an promising future for use in microscopic nuclear many-body theory.

\section{ACKNOWLEDGMENTS}

We wish to thank J. J. de Swart, J. L. Friar, T.-S. H. Lee, V. R. Pandharipande, B. Pudliner, and S. C. Pieper for many useful comments and stimulating discussions. During the early part of this project, R.B.W. enjoyed the hospitality and support of S. E. Koonin and the Kellogg Radiation Lab of the California Institute of Technology. The work of R.B.W. was supported by the U.S. Department of Energy, Nuclear Physics Division, under Contract No. W-31-109-ENG-38. The work of V.G.J.S. was supported by the Australian Research Council, while the work of R.S. was supported by the U.S. Department of Energy.
[1] R.B. Wiringa, R.A. Smith, and T.L. Ainsworth, Phys. Rev. C 29, 1207 (1984).

[2] I.E. Lagaris and V.R. Pandharipande, Nucl. Phys. A359, 331 (1981).

[3] R. Machleidt, K. Holinde, and Ch. Elster, Phys. Rep. 149, 1 (1987).

[4] R. Machleidt, Adv. Nucl. Phys. 19, 189 (1989).

[5] R.V. Reid, Jr., Ann. Phys. (N.Y.) 50, 411 (1968).

[6] M.M. Nagels, T.A. Rijken, and J.J. de Swart, Phys. Rev. D 17, 768 (1978).

[7] M. Lacombe, B. Loiseau, J.M. Richard, R. Vinh Mau, J. Côté, P. Pirès, and R. de Tourreil, Phys. Rev. C 21, 861 (1980).

[8] V. Stoks and J.J. de Swart, Phys. Rev. C 47, 761 (1993).

[9] Y. Wu, S. Ishikawa, and T. Sasakawa, Phys. Rev. Lett. 64, 1875 (1990); ibid. 66, 242 (1991).

[10] J.A. Nolen, Jr. and J.P. Schiffer, Annu. Rev. Nucl. Sci. 19, 471 (1969).

[11] R.B. Wiringa, Phys. Rev. C 43, 1585 (1991).

[12] S.C. Pieper, R.B. Wiringa, and V.R. Pandharipande, Phys. Rev. C 46, 1741 (1992).

[13] R.B. Wiringa, V. Fiks, and A. Fabrocini, Phys. Rev. C 38, 1010 (1988).

[14] V.G.J. Stoks, R.A.M. Klomp, C.P.F. Terheggen, and J.J. de Swart, Phys. Rev. C 49, 2950 (1994).

[15] J.R. Bergervoet, P.C. van Campen, R.A.M. Klomp, J.-L. de Kok, T.A. Rijken, V.G.J. Stoks, and J.J. de Swart, Phys. Rev. C 41, 1435 (1990).

[16] V.G.J. Stoks, R.A.M. Klomp, M.C.M. Rentmeester, and J.J. de Swart, Phys. Rev. C 48, 792 (1993).
[17] J.L. Friar, Ann. Phys. (N.Y.) 96, 158 (1976).

[18] G.J.M. Austen and J.J. de Swart, Phys. Rev. Lett. 50, 2039 (1983).

[19] V.G.J. Stoks and J.J. de Swart, Phys. Rev. C 41, 1235 (1990).

[20] G. Breit, Phys. Rev. 99, 1581 (1955).

[21] N. Auerbach, J. Hüfner, A.K. Kerman, and C.M. Shakin, Rev. Mod. Phys. 44, 48 (1972).

[22] J.L. Friar and J.W. Negele, Adv. Nucl. Phys. 8, 219 (1975).

[23] V.E. Krohn and G.R. Ringo, Phys. Rev. D 8, 1305 (1973).

[24] S. Platchkov et al., Nucl. Phys. A510, 740 (1990).

[25] E.M. Henley and G.A. Miller, in Mesons in Nuclei, edited by M. Rho and D. Wilkinson (North-Holland, Amsterdam, 1979), p. 406.

[26] V. Stoks, R. Timmermans, and J.J. de Swart, Phys. Rev. C 47, 512 (1993).

[27] I. Šlaus, Y. Akaishi, and H. Tanaka, Phys. Rep. 173, 257 (1989).

[28] G.F. de Téramond and B. Gabioud, Phys. Rev. C 36, 691 (1987).

[29] B. Gabioud et al., Phys. Rev. Lett. 42, 1508 (1979).

[30] O. Schori et al., Phys. Rev. C 35, 2252 (1987).

[31] R.A. Arndt, L.D. Roper, R.L. Workman, and M.W. McNaughton, Phys. Rev. D 45, 3995 (1992).

[32] J.R. Bergervoet, P.C. van Campen, W.A. van der Sanden, and J.J. de Swart, Phys. Rev. C 38, 15 (1988).

[33] D.V. Bugg and R.A. Bryan, Nucl. Phys. A540, 449 (1992). 
[34] R. Henneck, Phys. Rev. C 47, 1859 (1993).

[35] L. Koester and W. Nistler, Z. Phys. A 272, 189 (1975).

[36] P. Langacker and D.A. Sparrow, Phys. Rev. C 25, 1194 (1982); T.-S.H. Lee, private communication.

[37] C. van der Leun and C. Alderliesten, Nucl. Phys. A380, 261 (1982).

[38] I. Borbély, W. Grüebler, V. König, P.A. Schmelzbach, and A.M. Mukhamedzhanov, Phys. Lett. 160B, 17 (1985).

[39] N.L. Rodning and L.D. Knutson, Phys. Rev. C 41, 898 (1990).

[40] S. Klarsfeld, J. Martorell, J.A. Oteo, M. Nishimura, and D.W.L. Sprung, Nucl. Phys. A456, 373 (1986). This is the value for $r_{d}$ extracted from a reanalysis of the data on the experimental ratio of $(e, d)$ to $(e, p)$ scattering by R.W. Berard et al., Phys. Lett. 47B, 355 (1973), and Ref. [43].

[41] I. Lindgren, in Alpha-, Beta-, and Gamma-Ray Spectroscopy, edited by K. Siegbahn (North-Holland, Amsterdam, 1965), Vol. 2, p. 1620.

[42] D.M. Bishop and L.M. Cheung, Phys. Rev. A 20, 381 (1979); T.E.O. Ericson and M. Rosa-Clot, Nucl. Phys. A405, 497 (1983).

[43] G.G. Simon, Ch. Schmitt, and V.H. Walther, Nucl. Phys.
A364, 285 (1981).

[44] R. Cramer et al., Z. Phys. C 29, 513 (1985).

[45] R.G. Arnold et al., Phys. Rev. Lett. 35, 776 (1975).

[46] S. Auffret et al., Phys. Rev. Lett. 54, 649 (1985).

[47] R.G. Arnold et al., Phys. Rev. Lett. 58, 1723 (1987).

[48] M.E. Schulze et al., Phys. Rev. Lett. 52, 597 (1984).

[49] I. The et al., Phys. Rev. Lett. 67, 173 (1991).

[50] V.F. Dmitriev et al., Phys. Lett. 157B, 143 (1985).

[51] R. Gilman et al., Phys. Rev. Lett. 65, 1733 (1990).

[52] R. Schiavilla, V.R. Pandharipande, and D.O. Riska, Phys. Rev. C 40, 2294 (1989).

[53] R. Schiavilla and D.O. Riska, Phys. Rev. C 43, 437 (1991).

[54] R. Schiavilla, V.R. Pandharipande, and D.O. Riska, Phys. Rev. C 41, 309 (1990).

[55] J. Carlson, D.O. Riska, R. Schiavilla, and R.B. Wiringa, Phys. Rev. C 42, 830 (1990).

[56] G. Höhler et al., Nucl. Phys. B114, 505 (1976).

[57] J. Carlson, V.R. Pandharipande, and R. Schiavilla, in Modern Topics in Electron Scattering, edited by B. Frois and I. Sick (World Scientific, Singapore, 1991), p. 177.

[58] P.G. Blunden and D.O. Riska, Nucl. Phys. A536, 697 (1992).

[59] S.C. Pieper, private communication. 